\title{
ON A POWER OF $n$-ARY RELATIONAL SYSTEMS CARRIED BY STRONG HOMOMORPHISMS
}

\author{
N. PHROMMARAT
}

Received 11 July, 2017

\begin{abstract}
First, we introduce and discuss a new operation of product of $n$-ary relational systems which lies, as for generality, between their direct product and the direct product of their reflexive hulls. Then we study the power of $n$-ary relational systems obtained as the subsystem of their direct power carried by strong homomorphisms. We show that, with respect to the new operation of product, the power of $n$-ary relational systems studied satisfies week forms of the first and second exponential laws and, with respect to the direct product and direct sum, it satisfies the third exponential law.
\end{abstract}

2010 Mathematics Subject Classification: 08A02; 08A05; 08A99

Keywords: relational systems, direct product, strong homomorphism, the first, second and third exponential laws

\section{INTRODUCTION}

In his pioneering papers [2] and [3], G. Birkhoff introduced and studied the operation of a cardinal power of partially ordered sets and showed the validity of the first, second and third exponential laws for the operation, i.e.,

$\left(\mathbf{A}^{\mathbf{B}}\right)^{\mathbf{C}} \cong \mathbf{A}^{\mathbf{B} \times \mathbf{C}}$ - the first exponential law,

$\prod_{i \in I} \mathbf{A}_{i}^{\mathbf{B}} \cong\left(\prod_{i \in I} \mathbf{A}_{i}\right)^{\mathbf{B}}$ - the second exponential law, law.

$\prod_{i \in I} \mathbf{A}^{\mathbf{B}_{i}} \cong \mathbf{A}^{\sum_{i \in I} \mathbf{B}_{i}}$ (if $\mathbf{B}_{i}, i \in I$, are pair-wise disjoint) - the third exponential

Birkhoff's arithmetic of ordered sets has been generalized by several authors, see, e.g., [5] and [9]. The concept of a cardinal power was extended to relational systems in [7]. In this note, we will continue the study of the arithmetic of relational system.

In [8], M. Novotný and J. Šlapal introduced and studied new operations of product and power of relational systems which satisfy the first exponential law. The new product was defined by combining the direct (i.e., cardinal) operations of sum and product, and the new power was defined as the intersection of the direct power and another power of relational systems. In [1], František Bednařík and Josef Šlapal

This research was supported by Chiang Rai Rajabhat University, Thailand. 
studied the subsystem of the cardinal power of relational systems carried by the set of strong homomorphisms.

In the present paper, we will introduce a new operation of product of $n$-ary relational systems which generalizes the concept of combined product in [8]. Moreover, we will study the subsystem of the direct power carried by the set of strong homomorphisms. We will prove the validity of the third exponential law and weak forms of the first and second exponential laws.

\section{COMBINED PRODUCT OF $n$-ARY RELATIONAL SYSTEMS}

Throughout this paper, $n$ denotes a positive integer. By an $n$-ary relation $p$ on a set $A$ we mean a subset $p \subseteq A^{n}$. The pair $(A, p)$ is called an $n$-ary relational system.

Let $\mathbf{A}=(A, p), \mathbf{B}=(B, q)$ be a pair of $n$-ary relational systems. A mapping $f: B \rightarrow A$ is said to be a homomorphism of $\mathbf{B}$ into $\mathbf{A}$ if $\left(x_{1}, \ldots, x_{n}\right) \in q$ implies $\left(f\left(x_{1}\right), \ldots, f\left(x_{n}\right)\right) \in p$. A homomorphism $f$ of $\mathbf{B}=(B, q)$ into $\mathbf{A}=(A, p)$ is said to be a strong homomorphism of $\mathbf{B}$ into $\mathbf{A}$ if, for every $b_{1}, \ldots, b_{n} \in B$,

$\left(f\left(b_{1}\right), \ldots, f\left(b_{n}\right)\right) \in p$ implies $\left(b_{1}, \ldots, b_{n}\right) \in q$. We denote by $\operatorname{Hom}(\mathbf{B}, \mathbf{A})$ the set of all homomorphisms of $\mathbf{B}$ into $\mathbf{A}$ and by $\operatorname{SHom}(\mathbf{B}, \mathbf{A})$ the set of all strong homomorphisms of $\mathbf{B}$ into $\mathbf{A}$.

If $f: B \rightarrow A$ is a bijection and both $f: B \rightarrow A$ and $f^{-1}: A \rightarrow B$ are homomorphisms, then $f$ is called an isomorphism of $\mathbf{B}$ onto $\mathbf{A}$. If there exists an isomorphism of $\mathbf{B}$ onto $\mathbf{A}$, we say that $\mathbf{B}$ and $\mathbf{A}$ are isomorphic, in symbols $B \cong A$. An $n$-ary relational system $\mathbf{A}=(A, p)$ is said to be a subsystem of an $n$-ary relational system $\mathbf{B}=(B, q)$ if $A \subseteq B$ and $p=q \cap A^{n}$. Given a pair of $n$-ary relational systems $\mathbf{A}, \mathbf{B}$, we write $\mathbf{B} \preccurlyeq \mathbf{A}$ if $\mathbf{B}$ can be embedded into $\mathbf{A}$, i.e., if there is a relational subsystem $\mathbf{A}^{\prime}$ of $\mathbf{A}$ such that $\mathbf{B} \cong \mathbf{A}^{\prime}$.

The direct product of a family $\mathbf{A}_{i}=\left(A_{i}, p_{i}\right), i \in I$, of $n$-ary relational systems is the $n$-ary relational system $\prod_{i \in I} \mathbf{A}_{i}=\left(\prod_{i \in I} A_{i}, q\right)$ where $\prod_{i \in I} A_{i}$ is the cartesian product of sets and, for any $f_{1}, \ldots, f_{n} \in \prod_{i \in I} A_{i},\left(f_{1}, \ldots, f_{n}\right) \in q$ if and only if $\left(f_{1}(i), \ldots, f_{n}(i)\right) \in p_{i}$ for each $i \in I$. If the set $I$ is finite, say $I=\{1, \ldots, m\}$, then we write $\mathbf{A}_{1} \times \ldots \times \mathbf{A}_{m}$ instead of $\prod_{i \in I} \mathbf{A}_{i}$. If $\mathbf{A}_{i}=\mathbf{A}$ for each $i \in I$, then we write $\mathbf{A}^{I}$ instead of $\prod_{i \in I} \mathbf{A}_{i}$.

The direct sum of a family $\mathbf{A}_{i}=\left(A_{i}, p_{i}\right), i \in I$, of $n$-ary relational systems is the $n$-ary relational system $\sum_{i \in I} \mathbf{A}_{i}=\left(\bigcup_{i \in I} A_{i}, \bigcup_{i \in I} p_{i}\right)$. If the set $I$ is finite, say $I=\{1, \ldots, m\}$, then we write $\mathbf{A}_{1} \uplus \ldots \uplus \mathbf{A}_{m}$ instead of $\sum_{i \in I} \mathbf{A}_{i}$.

An $n$-ary relational system $\mathbf{A}=(A, p)$ is said to be reflexive provided that $\left(a_{1}, a_{2}, \ldots, a_{n}\right) \in p$ whenever $a_{1}=a_{2}=\ldots=a_{n}$. Let $\mathbf{A}=(A, p)$ be an $n$-ary relational system. We denote by $\bar{p}$ the $n$-ary relational system on $G$ such that, for every $a_{1}, \ldots, a_{n} \in A,\left(a_{1}, \ldots, a_{n}\right) \in \bar{p}$ if and only if $a_{1}=\ldots=a_{n}$ or $\left(a_{1}, \ldots, a_{n}\right) \in p$. The $n$-ary relational systems $(A, \bar{p})$ is called the reflexive hull of $\mathbf{A}$ and is denoted by $\overline{\mathbf{A}}$. 
Let $\mathbf{A}_{i}=\left(A_{i}, p_{i}\right), i \in I$, be a family of $n$-ary relational systems. The combined product of the family $\mathbf{A}_{i}, i \in I$, is the $n$-ary relational system $\bigotimes_{i \in I} \mathbf{A}_{i}=\left(\prod_{i \in I} A_{i}, r\right)$ given by $\bigotimes_{i \in I} \mathbf{A}_{i}=\sum_{i \in I} \prod_{j \in I} \mathbf{A}_{i j}$ where

$$
\mathbf{A}_{i j}= \begin{cases}\overline{\mathbf{A}}_{j} & \text { if } i=j, \\ \mathbf{A}_{j} & \text { if } i \neq j .\end{cases}
$$

Thus, for any $\left(a_{1}^{i} ; i \in I\right), \ldots,\left(a_{n}^{i} ; i \in I\right) \in \prod_{i \in I} \mathbf{A}_{i}$ we have $\left(a_{1}^{i} ; i \in I\right), \ldots,\left(a_{n}^{i} ; i \in\right.$ $I) \in r$ if and only if there exists a subset $J \subseteq I$, card $J \leq 1$, such that $\left(a_{1}^{i}, \ldots, a_{n}^{i}\right) \in p_{i}$ for every $i \in I \backslash J$ and $a_{1}^{i}=a_{2}^{i}=\ldots=a_{n}^{i}$ for every $i \in J$.

If the set $I$ is finite, say $I=\{1, \ldots, m\}$, we write $\mathbf{A}_{1} \otimes \ldots \otimes \mathbf{A}_{m}$ instead of $\bigotimes_{i \in I} A_{i}$. We then clearly have $\mathbf{A}_{1} \otimes \ldots \otimes \mathbf{A}_{m}=\left(\mathbf{A}_{11} \times \mathbf{A}_{12} \times \ldots \times \mathbf{A}_{1 m}\right) \uplus\left(\mathbf{A}_{21} \times\right.$ $\left.\mathbf{A}_{22} \times \ldots \times \mathbf{A}_{2 m}\right) \uplus \ldots \uplus\left(\mathbf{A}_{m 1} \times \mathbf{A}_{m 2} \ldots, \times \mathbf{A}_{m m}\right)=\left(\overline{\mathbf{A}}_{1} \times \mathbf{A}_{2} \times \ldots \times \mathbf{A}_{m}\right) \uplus\left(\mathbf{A}_{1} \times \overline{\mathbf{A}}_{2} \times\right.$ $\left.\ldots \times \mathbf{A}_{m}\right) \uplus \ldots \uplus\left(\mathbf{A}_{1} \times \mathbf{A}_{2} \times \ldots \times \overline{\mathbf{A}}_{m}\right)$.

In particular, if $I=\{1,2\}$, then, for any $\left(a_{1}, b_{1}\right), \ldots,\left(a_{n}, b_{n}\right) \in \mathbf{A}_{1} \times \mathbf{A}_{2}$, $\left(\left(a_{1}, b_{1}\right), \ldots,\left(a_{n}, b_{n}\right)\right) \in r$ if and only if one of the following three conditions is satisfied:

(i) $\left(a_{1}, \ldots, a_{n}\right) \in p_{1}$ and $\left(b_{1}, \ldots, b_{n}\right) \in p_{2}$,

(ii) $a_{1}=a_{2}=\ldots=a_{n}$ and $\left(b_{1}, \ldots, b_{n}\right) \in p_{2}$,

(iii) $\left(a_{1}, \ldots, a_{n}\right) \in p_{1}$ and $b_{1}=b_{2}=\ldots=b_{n}$.

Example 1. Let $\mathbf{A}=(A, p), \mathbf{B}=(B, q)$ be binary relational systems where $A=$ $\{1,2\}, B=\{a, b\}, p=\{(1,2)\}$ and $q=\{(a, b)\}$. Then $\mathbf{A} \otimes \mathbf{B}=(A \times B, r)$ where $r=$ $\{((1, a),(2, b)),((1, a),(1, b)),((2, a),(2, b)),((1, a),(2, a)),((1, b),(2$, b)) $\}$.

Example 2. Let $\mathbf{A}=(A, p), \mathbf{B}=(B, q)$ be binary relational systems where $A=$ $\{1,2\}, B=\{a\}, p=\{(1,2)\}$ and $q=\{(a, a)\}$. Then $\mathbf{A} \otimes \mathbf{B}=(A \times B, r)$ where $r=$ $\{((1, a),(2, a)),((1, a),(1, a)),((2, a),(2, a))\}$. Thus, $\mathbf{A} \otimes \mathbf{B}$ is reflexive.

Remark 1. Let $\mathbf{A}_{i}, i \in I$, be a family of $n$-ary relational systems. If $\mathbf{A}_{i}$ is reflexive for every $i \in I$, then $\prod_{i \in I} \mathbf{A}_{i}=\bigotimes_{i \in I} \mathbf{A}_{i}$. If at most one of the $n$-ary relational systems $\mathbf{A}_{i}, i \in I$, is not reflexive, then $\bigotimes_{i \in I} \mathbf{G}_{i}$ is reflexive.

First, we will show that the combined product of $n$-ary relational systems distributes over their direct sum.

Proposition 1. Let $\mathbf{A}=(A, p)$ and $\mathbf{B}_{\mathbf{i}}=\left(B_{i}, q_{i}\right), i \in I$, be $n$-ary relational systems. Then $\mathbf{A} \otimes \sum_{i \in I} \mathbf{B}_{i}=\sum_{i \in I}\left(\mathbf{A} \otimes \mathbf{B}_{i}\right)$.

Proof. Let $\sum_{i \in I} \mathbf{B}_{i}=\left(\bigcup_{i \in I} B_{i}, s\right), \mathbf{A} \otimes \mathbf{B}_{i}=\left(A \times B_{i}, r_{i}\right)$ for each $i \in I, \mathbf{A} \otimes$ $\sum_{i \in I} \mathbf{B}_{i}=\left(A \times \bigcup_{i \in I} B_{i}, u\right)$ and $\sum_{i \in I}\left(\mathbf{A} \otimes \mathbf{B}_{i}\right)=\left(\bigcup_{i \in I}\left(A \times B_{i}\right), v\right)$. We will show that, for every

$$
\left(a_{1}, b_{1}\right), \ldots,\left(a_{n}, b_{n}\right) \in A \times \bigcup_{i \in I} B_{i}=\bigcup_{i \in I}\left(A \times B_{i}\right),\left(\left(a_{1}, b_{1}\right), \ldots,\left(a_{n}, b_{n}\right)\right) \in u
$$


if and only if $\left(\left(a_{1}, b_{1}\right), \ldots,\left(a_{n}, b_{n}\right)\right) \in v$. It is easy to see that the following five conditions are equivalent:

(1) $\left(\left(a_{1}, b_{1}\right), \ldots,\left(a_{n}, b_{n}\right)\right) \in u$;

(2) one of the following three cases occurs:

(i) $\left(a_{1}, \ldots, a_{n}\right) \in p$ and $\left(b_{1}, \ldots, b_{n}\right) \in s$,

(ii) $a_{1}=a_{2}=\ldots=a_{n}$ and $\left(b_{1}, \ldots, b_{n}\right) \in s$,

(iii) $\left(a_{1}, \ldots, a_{n}\right) \in p$ and $b_{1}=b_{2}=\ldots=b_{n}$;

(3) one of the following three cases occurs:

(i) $\left(a_{1}, \ldots, a_{n}\right) \in p$ and $\left(b_{1}, \ldots, b_{n}\right) \in q_{i}$ for some $i \in I$,

(ii) $a_{1}=a_{2}=\ldots=a_{n}$ and $\left(b_{1}, \ldots, b_{n}\right) \in q_{i}$ for some $i \in I$,

(iii) $\left(a_{1}, \ldots, a_{n}\right) \in p$ and $b_{1}=b_{2}=\ldots=b_{n}$;

(4) $\left(\left(a_{1}, b_{1}\right), \ldots,\left(a_{n}, b_{n}\right)\right) \in r_{i}$ for some $i \in I$;

(5) $\left(\left(a_{1}, b_{1}\right), \ldots,\left(a_{n}, b_{n}\right)\right) \in v$.

This proves the statement.

\section{POWER OF RELATIONAL SYSTEMS With RESPECT TO STRONG HOMOMORPHISMS}

Definition 1. Let $\mathbf{B}=(B, q)$ and $\mathbf{A}=(A, p)$ be $n$-ary relational systems. The direct power of $\mathbf{A}$ and $\mathbf{B}$ is the $n$-ary relational system $(\operatorname{Hom}(\mathbf{B}, \mathbf{A}), r)$ where, for any $f_{1}, \ldots, f_{n} \in \operatorname{Hom}(\mathbf{B}, \mathbf{A}), \quad\left(f_{1}, \ldots, f_{n}\right) \in r$ if and only if $\left(f_{1}(x), \ldots\right.$, $\left.f_{n}(x)\right) \in p$ for each $x \in B$. The subsystem $\left(\operatorname{SHom}(\mathbf{B}, \mathbf{A}), r \cap(\operatorname{SHom}(\mathbf{B}, \mathbf{A}))^{n}\right)$ of the direct power $(\operatorname{Hom}(\mathbf{B}, \mathbf{A}), r)$ of $\mathbf{A}$ and $\mathbf{B}$ is called the direct power of $\mathbf{A}$ and $\mathbf{B}$ with respect to strong homomorphisms.

For any pair of $n$-ary relational systems $\mathbf{A}, \mathbf{B}$, we denote by $\mathbf{A}^{\mathbf{B}}$ and $\mathbf{A}^{\diamond \mathbf{B}}$ the direct power and the direct power with respect to strong homomorphisms, respectively, of $\mathbf{A}$ and $\mathbf{B}$.

It is easy to see that the direct power $\mathbf{A}^{\mathbf{B}}$ of $n$-ary relational systems is reflexive whenever $\mathbf{A}$ is reflexive.

Example 3. Let $\mathbf{A}=(A, p), \mathbf{B}=(B, q)$ be binary relational systems where $A=$ $\{1,2\}, B=\{a\}, p=A \times A$ and $q=\{(a, a)\}$. Assume that $f: A \rightarrow B$ is a map given by $f(1)=f(2)=a$. Then, $\operatorname{Hom}(\mathbf{A}, \mathbf{B})=\operatorname{SHom}(\mathbf{A}, \mathbf{B})=\{f\}$ and $\mathbf{A}^{\diamond \mathbf{B}}=$ $(\operatorname{SHom}(\mathbf{B}, \mathbf{A}), s)$ where $s=\{(f, f)\}$.

Example 4. Let $\mathbf{A}=(A, p), \mathbf{B}=(B, q)$ be binary relational systems where $A=$ $\{1,2,3\}, B=\{a, b\}, p=\{(1,1),(2,2),(3,3),(1,2),(2,1),(1,3),(3,1),(2,3)\}$ and $q=$ $B \times B$. Then, every map of $A$ into $B$ is a homomorphism of $A$ into $B$. Assume that $f, g: A \rightarrow B$ are maps given by $f(1)=f(2)=a, f(3)=b$ and $g(1)=g(3)=$ $a, g(2)=b$. Then, $\operatorname{SHom}(\mathbf{A}, \mathbf{B})=\{f, g\}$ and $\mathbf{A}^{\diamond \mathbf{B}}=(\operatorname{SHom}(\mathbf{B}, \mathbf{A}), s)$ where $s=$ $\operatorname{SHom}(\mathbf{A}, \mathbf{B}) \times \operatorname{SHom}(\mathbf{A}, \mathbf{B})$. 
We will show now that, for the combined product of $n$-ary relational systems and their power with respect to strong homomorphisms, weak forms of the first and second exponential laws hold.

Theorem 1. Let $\mathbf{A}, \mathbf{B}, \mathbf{C}$ be n-ary relational systems. Then

$$
\mathbf{A}^{\diamond(\mathbf{B} \otimes \mathbf{C})} \preccurlyeq\left(\mathbf{A}^{\diamond \mathbf{B}}\right)^{\diamond \mathbf{C}}
$$

Proof. Let $\mathbf{A}=(A, q), \mathbf{B}=(B, p), \mathbf{C}=(C, s), \mathbf{A}^{\diamond \mathbf{B}}=(\operatorname{SHom}(\mathbf{B}, \mathbf{A}), r)$,

$\mathbf{B} \otimes \mathbf{A}=(B \times A, v),\left(\mathbf{A}^{\diamond \mathbf{B}}\right)^{\diamond \mathbf{C}}=(\operatorname{SHom}(\mathbf{C}, \operatorname{SHom}(\mathbf{B}, \mathbf{A})), t)$ and $\mathbf{A}^{\diamond(\mathbf{B} \otimes \mathbf{C})}=$ $(\operatorname{SHom}(\mathbf{B} \otimes \mathbf{C}, \mathbf{A}), u)$ be $n$-ary relational systems.

We define a map $\alpha: \operatorname{SHom}(\mathbf{B} \otimes \mathbf{C}, \mathbf{A}) \rightarrow \operatorname{SHom}(\mathbf{C}, \operatorname{SHom}(\mathbf{B}, \mathbf{A}))$ by $\alpha(h)(c)(b)=$ $h(b, c)$ whenever $h \in \operatorname{SHom}(\mathbf{B} \otimes \mathbf{C}, \mathbf{A}), c \in C$ and $b \in B$.

Let $h \in \operatorname{SHom}(\mathbf{B} \otimes \mathbf{C}, \mathbf{A}), c \in C$ and $\left(b_{1}, \ldots, b_{n}\right) \in q$. Consequently, $\left(\left(b_{1}, c\right), \ldots,\left(b_{n}, c\right)\right) \in v$. Since $h$ is a homomorphism, we have $\left(h\left(b_{1}, c\right), \ldots\right.$, $\left.h\left(b_{n}, c\right)\right) \in p$. Thus, $\left(\alpha(h)(c)\left(b_{1}\right), \ldots, \alpha(h)(c)\left(b_{n}\right)\right)=\left(h\left(b_{1}, c\right), \ldots, h\left(b_{n}, c\right)\right) \in p$. Therefore, $\alpha(h)(c) \in \operatorname{Hom}(\mathbf{B}, \mathbf{A})$ for each $c \in C$.

Let $b_{1}, \ldots, b_{n} \in B$ and $\left(\alpha(h)(c)\left(b_{1}\right), \ldots, \alpha(h)(c)\left(b_{n}\right)\right) \in p$. Then $\left(\alpha(h)(c)\left(b_{1}\right)\right.$, $\left.\ldots, \alpha(h)(c)\left(b_{n}\right)\right)=\left(h\left(b_{1}, c\right), \ldots, h\left(b_{n}, c\right)\right) \in p$. Since $h$ is a strong homomorphism, we get $\left(\left(b_{1}, c\right), \ldots,\left(b_{n}, c\right)\right) \in v$. Thus, $\left(b_{1}, \ldots, b_{n}\right) \in q$. Then, $\alpha(h)(c) \in \operatorname{SHom}(\mathbf{B}, \mathbf{A})$ for each $c \in C$.

Next, let $\left(c_{1}, \ldots, c_{n}\right) \in s$. Then $\left(\left(b, c_{1}\right), \ldots,\left(b, c_{n}\right)\right) \in v$. Since $h$ is a homomorphism, we get $\left(h\left(b, c_{1}\right), \ldots, h\left(b, c_{n}\right)\right) \in p$. So $\left(\alpha(h)(b)\left(c_{1}\right), \ldots, \alpha(h)(b)\left(c_{n}\right)\right)$ $=\left(h\left(b, c_{1}\right), \ldots, h\left(b, c_{n}\right)\right) \in p$. Consequently, $\left(\alpha(h)\left(c_{1}\right), \ldots, \alpha(h)\left(c_{n}\right)\right) \in r$. Hence, $\alpha(h) \in \operatorname{Hom}(\mathbf{C}, \operatorname{Hom}(\mathbf{B}, \mathbf{A}))$.

Let $c_{1}, \ldots, c_{n} \in C$ and $\left(\alpha(h)\left(c_{1}\right), \ldots, \alpha(h)\left(c_{n}\right)\right) \in r$. Then $\left(\alpha(h)\left(c_{1}\right) b, \ldots\right.$, $\left.\alpha(h)\left(c_{n}\right) b\right) \in p$, hence $\left(\alpha(h)(b)\left(c_{1}\right), \ldots, \alpha(h)(b)\left(c_{n}\right)\right)=\left(h\left(b, c_{1}\right), \ldots, h\left(b, c_{n}\right)\right) \in p$. Now, as $h$ is a strong homomorphism, it follows that $\left(\left(b, c_{1}\right), \ldots,\left(b, c_{n}\right)\right) \in v$. Hence $\left(c_{1}, \ldots, c_{n}\right) \in s$. Therefore, $\alpha(h) \in \operatorname{SHom}(\mathbf{C}, \operatorname{Hom}(\mathbf{B}, \mathbf{A}))$.

We will show that $\alpha$ is a homomorphisms. Let $\left(h_{1}, \ldots, h_{n}\right) \in u,\left(c_{1}, \ldots, c_{n}\right) \in s$ and $\left(b_{1}, \ldots, b_{n}\right) \in q$. Then we have $\left(\left(b_{1}, c_{1}\right), \ldots,\left(b_{n}, c_{n}\right)\right) \in v$ and, consequently, $\left(h_{1}\left(b_{1}, c_{1}\right), \ldots, h_{n}\left(b_{n}, c_{n}\right)\right) \in p . \quad$ Therefore, $\quad\left(\alpha\left(h_{1}\right)\left(c_{1}\right)\left(b_{1}\right), \ldots\right.$, $\left.\alpha\left(h_{n}\right)\left(c_{n}\right)\left(b_{n}\right)\right)=\left(h_{1}\left(b_{1}, c_{1}\right), \ldots, h_{n}\left(b_{n}, c_{n}\right)\right) \in p$. Hence, $\quad\left(\alpha\left(h_{1}\right)\left(c_{1}\right), \ldots\right.$, $\left.\alpha\left(h_{n}\right)\left(c_{n}\right)\right) \in r$, so that $\left(\alpha\left(h_{1}\right), \ldots, \alpha\left(h_{n}\right)\right) \in t$. Thus, $\alpha$ is a homomorphism.

Finally, we will show that $\alpha$ is an injection. Let $\alpha\left(h_{1}\right)=\alpha\left(h_{2}\right)$ for a pair $h_{1}, h_{2} \in$ $\operatorname{SHom}(\mathbf{B} \otimes \mathbf{C}, \mathbf{A})$. Then $\alpha\left(h_{1}\right) c=\alpha\left(h_{2}\right) c$ for every $c \in C$ and we have $\alpha\left(h_{1}\right)(c) b=$ $\alpha\left(h_{2}\right)(c) b$ for every $c \in C$ and $b \in B$. Hence $h_{1}(b, c)=h_{2}(b, c)$ for every $(b, c) \in$ $B \times C$. Thus, $h_{1}=h_{2}$ and we have shown that $\alpha$ is an injection.

Therefore $\alpha$ is an embedding of $\mathbf{A}^{\diamond(\mathbf{B} \otimes \mathbf{C})}$ into $\left(\mathbf{A}^{\diamond \mathbf{B}}\right)^{\diamond \mathbf{C}}$ and the proof is complete. 
Theorem 2. Let $\mathbf{A}_{i}=\left(A_{i}, p_{i}\right), i \in I$, be a family of $n$-ary relational systems and $\mathbf{B}$ be an $n$-ary relational system. Then

$$
\bigotimes_{i \in I}\left(\mathbf{A}_{i}^{\diamond \mathbf{B}}\right) \preccurlyeq\left(\bigotimes_{i \in I} \mathbf{A}_{i}\right)^{\diamond \mathbf{B}}
$$

Proof. Let $\mathbf{A}_{i}=\left(A_{i}, p_{i}\right)$ for every $i \in I, \mathbf{B}=(B, q), \bigotimes_{i \in I} \mathbf{A}_{i}=\left(\prod_{i \in I} A_{i}, r\right)$, $\mathbf{A}_{i}^{\diamond B}=\left(\operatorname{SHom}\left(\mathbf{B}, \mathbf{A}_{i}\right), u_{i}\right)$ for every $i \in I, \bigotimes_{i \in I} \mathbf{A}_{i}^{\diamond \mathbf{B}}=\left(\prod_{i \in I} \operatorname{SHom}\left(\mathbf{B}, \mathbf{A}_{i}\right)\right.$, $s)$ and $\left(\bigotimes_{i \in I} \mathbf{A}_{i}\right)^{\diamond \mathbf{B}}=\left(\operatorname{Hom}\left(\mathbf{B}, \bigotimes_{i \in I} \mathbf{A}_{i}\right), t\right)$.

We define a map $\alpha: \prod_{i \in I} \operatorname{SHom}\left(\mathbf{B}, \mathbf{A}_{i}\right) \rightarrow\left(\prod_{i \in I} A_{i}\right)^{H}$ by $\alpha\left(f^{i} ; i \in I\right)(b)=$ $\left(f^{i}(b) ; i \in I\right)$ for each $b \in B$.

Let $\left(f^{i} ; i \in I\right) \in \prod_{i \in I} \operatorname{SHom}\left(\mathbf{B}, \mathbf{A}_{i}\right)$ and $\left(b_{1}, \ldots, b_{n}\right) \in q$. Since $f^{i} \in \operatorname{Hom}\left(\mathbf{B}, \mathbf{A}_{i}\right)$, we have $\left(f^{i}\left(b_{1}\right), \ldots, f^{i}\left(b_{n}\right)\right) \in p_{i}$ for every $i \in I$. Then $\left(\left(f^{i}\left(b_{1}\right) ; i\right.\right.$

$\left.\in I), \ldots,\left(f^{i}\left(b_{n}\right) ; i \in I\right)\right) \in r$ and we have $\left(\alpha\left(f^{i} ; i \in I\right)\left(b_{1}\right), \ldots, \alpha\left(f^{i} ; i \in I\right)\left(b_{n}\right)\right) \in$ $r$. Therefore, $\alpha\left(f^{i} ; i \in I\right) \in \operatorname{Hom}\left(\mathbf{B}, \bigotimes_{i \in I} \mathbf{A}_{i}\right)$.

Let $\left(f^{i} ; i \in I\right) \in \prod_{i \in I} \operatorname{SHom}\left(\mathbf{B}, \mathbf{A}_{i}\right)$ and

$$
\left(\alpha\left(f^{i} ; i \in I\right)\left(b_{1}\right), \ldots, \alpha\left(f^{i} ; i \in I\right)\left(b_{n}\right)\right) \in r .
$$

Consequently, $\left(\left(f^{i}\left(b_{1}\right) ; i \in I\right), \ldots,\left(f^{i}\left(b_{n}\right) ; i \in I\right)\right) \in r$. Therefore, $\left(f^{i}\left(b_{1}\right), \ldots, f^{i}\left(b_{n}\right)\right) \in p_{i}$ for every $i \in I$. As $f^{i}$ is a strong homomorphism for every $i \in I$, we have $\left(b_{1}, \ldots, b_{n}\right) \in q$. We have shown that $\alpha$ maps $\prod_{i \in I} \operatorname{SHom}\left(\mathbf{B}, \mathbf{A}_{i}\right)$ into $\operatorname{SHom}\left(\mathbf{B}, \bigotimes_{i \in I} \mathbf{A}_{i}\right)$.

We will show that $\alpha$ is a homomorphism. Let $\left(f_{1}^{i} ; i \in I\right), \ldots,\left(f_{n}^{i} ; i \in I\right) \in$ $\prod_{i \in I} \operatorname{SHom}\left(\mathbf{B}, \mathbf{A}_{i}\right)$ and $\left(\left(f_{1}^{i} ; i \in I\right), \ldots,\left(f_{n}^{i} ; i \in I\right)\right) \in s$. Let $J \subseteq I$, card $J \leq 1$. Consequently, $\left(f_{1}^{i}, \ldots, f_{n}^{i}\right) \in u_{i}$ for every $i \in I \backslash J$ and $f_{1}^{i}=f_{2}^{i}=\ldots=f_{n}^{i}$ for every $i \in J$. Hence, $\left(f_{1}^{i}(b), \ldots, f_{n}^{i}(b)\right) \in p_{i}$ for every $i \in I \backslash J$ and every $b \in$ $B$ and $f_{1}^{i}(b)=f_{2}^{i}(b)=\ldots=f_{n}^{i}(b)$ for every $i \in J$ and every $b \in B$. Hence, $\left(\left(f_{1}^{i}(b) ; i \in I\right), \ldots,\left(f_{n}^{i}(b) ; i \in I\right)\right) \in r$ for every $b \in B$. Therefore, $\left(\alpha\left(f_{1}^{i} ; i \in\right.\right.$ $\left.I)(b), \ldots, \alpha\left(f_{n}^{i} ; i \in I\right)(b)\right) \in r$ for every $b \in B$ and then $\left(\alpha\left(f_{1}^{i} ; i \in I\right), \ldots, \alpha\left(f_{n}^{i} ; i \in\right.\right.$ $I)) \in t$. Thus, $\alpha$ is a homomorphism of $\bigotimes_{i \in I}\left(\mathbf{A}_{i}^{\diamond \mathbf{B}}\right)$ into $\left(\bigotimes_{i \in I} \mathbf{A}_{i}\right)^{\diamond \mathbf{B}}$.

Suppose that $\alpha\left(f^{i} ; i \in I\right)=\alpha\left(g^{i} ; i \in I\right)$ where $\left(f^{i} ; i \in I\right),\left(g^{i} ; i \in I\right) \in$ $\prod_{i \in I} \operatorname{SHom}\left(\mathbf{B}, \mathbf{A}_{i}\right)$. Then $\left(f^{i}(b) ; i \in I\right)=\alpha\left(f^{i} ; i \in I\right)(b)=\alpha\left(g^{i} ; i \in I\right)(b)=$ $\left(g^{i}(b) ; i \in I\right)$ for every $b \in B$. Therefore, $f^{i}(b)=g^{i}(b)$ for every $i \in I$ and every $b \in B$. Hence, $f^{i}=g^{i}$ for every $i \in I$. Thus, $\alpha$ : $\prod_{i \in I} \operatorname{SHom}\left(\mathbf{B}, \mathbf{A}_{i}\right) \rightarrow$ $\operatorname{SHom}\left(\mathbf{B}, \bigotimes_{i \in I} \mathbf{A}_{i}\right)$ is an injection. We have shown that $\bigotimes_{i \in I}\left(\mathbf{A}_{i}^{\diamond \mathbf{B}}\right) \preccurlyeq\left(\bigotimes_{i \in I} \mathbf{A}_{i}\right)^{\diamond \mathbf{B}}$.

Next, we will show that, for the powers of $n$-ary relational systems with respect to strong homomorphisms, the third exponential law holds.

Theorem 3. Let $\mathbf{A}$ be an $n$-ary relational system and let $\mathbf{B}_{i}, i \in I$, be a family of pair-wise disjoint $n$-ary relational systems. Then 


$$
\prod_{i \in I} \mathbf{A}^{\diamond \mathbf{B}_{i}} \cong \mathbf{A}^{\diamond \sum_{i \in I} \mathbf{B}_{i}}
$$

Proof. Let $\mathbf{A}=(A, p), \mathbf{B}_{i}=\left(B_{i}, q_{i}\right)$ for every $i \in I, \sum_{i \in I} \mathbf{B}_{i}=\left(\sum_{i \in I} B_{i}, v\right)$, $\mathbf{A}^{\diamond \mathbf{B}_{\mathbf{i}}}=\left(\operatorname{SHom}\left(\mathbf{B}_{i}, \mathbf{A}\right), r_{i}\right)$ for every $i \in I, \prod_{i \in I} \mathbf{A}^{\diamond \mathbf{B}_{i}}=\left(\prod_{i \in I} \operatorname{SHom}\left(\mathbf{B}_{i}, \mathbf{A}\right)\right.$, $t)$ and $\mathbf{A}^{\diamond \sum_{i \in I} \mathbf{B}_{i}}=\left(\operatorname{SHom}\left(\sum_{i \in I} \mathbf{B}_{i}, \mathbf{A}\right), u\right)$.

We define a map $\varphi: \prod_{i \in I} \operatorname{SHom}\left(\mathbf{B}_{i}, \mathbf{A}\right) \rightarrow \operatorname{SHom}\left(\sum_{i \in I} \mathbf{B}_{i}, \mathbf{A}\right)$ by $\varphi\left(f^{i} ; i \in\right.$ $I)=h$ where $h(t)=f^{i}(t)$ whenever $t \in H_{i}$ (and $\left.i \in I\right)$. Let $\left(b_{1}, \ldots, b_{n}\right) \in q_{i}$ for any $i \in I$. Since $f_{i}$ is a homomorphism, we have $\left(\varphi\left(f^{i} ; i \in I\right) b_{1}, \ldots,\left(f^{i} ; i \in\right.\right.$ $\left.I) b_{n}\right)=\left(h\left(b_{1}\right), \ldots,\left(h\left(b_{n}\right)\right)=\left(f^{i}\left(b_{1}\right), \ldots,\left(f^{i}\left(b_{n}\right)\right)\right.\right.$ $\in r$ for any $i \in I$. Then $\varphi\left(f^{i} ; i \in I\right) \in \operatorname{Hom}\left(\sum_{i \in I} \mathbf{B}_{i}, A\right)$.

Let $\left(\varphi\left(f^{i} ; i \in I\right) b_{1}, \ldots,\left(f^{i} ; i \in I\right) b_{n}\right)$ for every $b_{1}, \ldots, b_{n} \in B$. Thus, $\left(\left(f^{i}\left(b_{1}\right), \ldots,\left(f^{i}\left(b_{n}\right)\right)=\left(h\left(b_{1}\right), \ldots,\left(h\left(b_{n}\right)\right)=\varphi\left(f^{i} ; i \in I\right) b_{1}, \ldots,\left(f^{i} ; i \in I\right) b_{n}\right) \in\right.\right.$ $r$ for any $i \in I$. Since $f^{i}$ is a strong homomorphism, we have $\left(b_{1}, \ldots, b_{n}\right) \in q_{i}$ for any $i \in I$. Therefore, $\varphi\left(f^{i} ; i \in I\right) \in \operatorname{SHom}\left(\sum_{i \in I} \mathbf{B}_{i}, A\right)$.

Further, we define a map $\alpha: \operatorname{SHom}\left(\sum_{i \in I} \mathbf{B}_{i}, \mathbf{A}\right) \rightarrow \prod_{i \in I} \operatorname{SHom}\left(\mathbf{B}_{i}, \mathbf{A}\right)$ by $\alpha(h)=\left(f^{i} ; \quad i \in I\right)$ whenever $h \in \operatorname{SHom}\left(\sum_{i \in I} \mathbf{B}_{i}, \mathbf{A}\right)$ where $f^{i}=\left.h\right|_{B_{i}}$ for every $i \in I$. It is easy to see that $f_{i}$ is a strong homomorphism of $\mathbf{B}_{i}$ into $\mathbf{A}$ for every $i \in I$. It follows that $\alpha(h) \in \prod_{i \in I} \operatorname{SHom}\left(\mathbf{B}_{i}, \mathbf{A}\right)$.

We will show that both $\varphi$ and $\alpha$ are homomorphisms. Let $\left(f_{1}^{i} ; i \in I\right), \ldots$, $\left(f_{n}^{i} ; i \in I\right) \in \prod_{i \in I} \operatorname{SHom}\left(\mathbf{B}_{i}, \mathbf{A}\right)$ and $\left(\left(f_{1}^{i} ; i \in I\right), \ldots,\left(f_{n}^{i} ; i \in I\right)\right) \in T$. Consequently, $\left(f_{1}^{i}, \ldots, f_{n}^{i}\right) \in r_{i}$ for each $i \in I$. Then

$$
\left(f_{1}^{i}(b), \ldots, f_{n}^{i}(b)\right)=\left(h_{1}(b), \ldots, h_{n}(b)\right) \in p
$$

for each $i \in I$ and $b \in \sum_{i \in I} B_{i}$. Therefore, $\left(\varphi\left(f_{1}^{i} ; i \in I\right), \ldots, \varphi\left(f_{n}^{i} ; i \in I\right)\right) \in u$. Thus, $\varphi$ is a homomorphism.

Let $h_{1}, \ldots, h_{n} \in \operatorname{SHom}\left(\sum_{i \in I} \mathbf{B}_{i}, \mathbf{A}\right)$ and $\left(h_{1}, \ldots, h_{n}\right) \in U$. Then $\left(\left.h_{1}\right|_{B_{i}}(b)\right.$, $\left.\ldots,\left.h_{n}\right|_{B_{i}}(b)\right)=\left(f_{1}^{i}(b), \ldots, f_{n}^{i}(b)\right) \in p$ for any $i \in I$ and $b \in \sum_{i \in I} B_{i}$. Therefore, $\left(f_{1}^{i}, \ldots, f_{n}^{i}\right) \in r_{i}$. Thus, $\left(\left(f_{1}^{i} ; i \in I\right), \ldots,\left(f_{n}^{i} ; i \in I\right)\right) \in T$ and then

$\left(\alpha\left(h_{1}\right), \ldots, \alpha\left(h_{n}\right)\right) \in t$.

It follows that $\varphi$ is an isomorphism with $\varphi^{-1}=\alpha$.

Remark 2. An n-ary hyperalgebra is an $(n+1)$-ary relational system $\mathbf{G}=(G, p)$ such that, for any $x_{1}, \ldots, x_{n} \in G$, there exists $y \in G$ with $\left(x_{1}, \ldots, x_{n}, y\right) \in p$ (cf. [10]). The $(n+1)$-ary relation $p$ on $\mathbf{G}$ may then be considered to be a map $p: G^{n} \rightarrow \exp$ $G$ and it is called an $n$-ary hyperoperation on $G$; we write $y \in p\left(x_{1}, \ldots, x_{n}\right)$ instead of $\left(x_{1}, \ldots, x_{n}, y\right) \in p$. If such $y$ is unique whenever $x_{1}, \ldots, x_{n} \in G$, then the $(n+1)$ ary relational system $\mathbf{G}$ is nothing but an $n$-ary algebra (cf. [6]). The $(n+1)$-ary relation $p$ may then be considered to be a map $p: G^{n} \rightarrow G$ and it is called an $n$-ary operation on $G$; we write $y=p\left(x_{1}, \ldots, x_{n}\right)$ instead of $\left(x_{1}, \ldots, x_{n}, y\right) \in p$. Powers of hyperalgebras were studied in [10] and those of algebras in [4]. Of course, if 
$\mathbf{A}, \mathbf{B}$ are $n$-ary algebras (hyperalgebras), then the power $\mathbf{A}^{\diamond \mathbf{B}}$ need not be an $n$-ary algebra (hyperalgebra). So, it is an open problem to find conditions under which the three exponential laws or at least their week forms are satisfied for $n$-ary algebras (hyperalgebras).

\section{REFERENCES}

[1] F. Bednařík and J. Šlapal, "Exponentiation of relational system with respect to strong homomorphism." Math. Pannonica, vol. 10, no. 2, pp. 167-175, 1999.

[2] G. Birkhoff, "An exteneded arithmetic." Duke Math. J., vol. 3, no. 2, pp. 311-316, 1937, doi: 10.1215/S0012-7094-37-00323-5.

[3] G. Birkhoff, "Generalized arithmetic.” Duke Math. J., vol. 9, no. 2, pp. 283-302, 1942, doi: 10.1215/S0012-7094-42-00921-9.

[4] N. Chaisansuk and S. Leeratanavalee, "On powers of relational and algebraic systems." Acta Math.Hungar., vol. 139, no. 3, pp. 195-207, 2013, doi: 10.1007/s10474-012-0257-9.

[5] M. M. Day, "Arithmetic of ordered systems." Transact. Amer. Math. Soc., vol. 58, no. 1, pp. 1-43, 1945, doi: 10.2307/1990233.

[6] G. Grätzer, Universal Algebra. Springer-Verlag, New York-Heidelberg-Berlin, $1979 . \quad$ doi: 10.1007/978-0-387-77487-9.

[7] V. Novák, "On a power of relational structures." Czech. Math. J., vol. 35, pp. 321-328, 1985.

[8] M. Novotný and J. Šlapal, "On powers of relational structures." Publ. Math. Debrecen., vol. 61, pp. 341-356, 2002.

[9] J. Šlapal, "Direct arithmetics of relational systems." Publ. Math. Debrecen., no. 38, pp. 39-48, 1991.

[10] J. Šlapal, “On exponentiation of universal hyperalgebras.” Alg. Universalis, no. 44, pp. 187-193, 2000, doi: 10.1007/s00012005180.

Author's address

N. Phrommarat

Department of Mathematics, Faculty of Science and Technology, Chiang Rai Rajabhat University, 57100 Chiang Rai, Thailand

E-mail address: nitimachaisansuk@gmail.com 